\title{
Bilateral Kimura's disease with devastating visual outcome
}

\begin{abstract}
Kimura's disease is a chronic inflammatory disease involving connective tissues. We treated a patient with bilateral periocular Kimura's disease causing eyelid deformity and exophthalmos for over 20 years. She has been operated on 23 times and biopsies were taken and studied histologically. The disease continued despite the multiple surgical excisions. The consequent exophthalmos and lid changes caused lagophthalmos that resulted in severe visual impairment due to chronic bilateral corneal exposure. Kimura's disease may have a devastating effect on visual function.
\end{abstract}

Key words Kimura's disease, ALHE

\section{Case report}

A 54-year-old Caucasian woman, a new immigrant from Russia, suffered from bilateral swollen eyelids and proptosis from the age of 30 years. She had undergone 20 eyelid operations in Russia up to 1993. She first presented to us with bilateral exophthalmos, lagophthalmos, swollen eyelids with small nodules and dark pigmentation of the eyelid skin. The patient was unable to close her eyes due to severe proptosis and stiffness of the lower lid with diminished movement. Infiltration of orbicularis was not ruled out by biopsy. Her visual acuity in the right eye was $6 / 12$, and finger counting in the left eye due to corneal opacities. An orbital CT scan showed diffuse enlargement of the extraocular muscles including their tendons and diffuse infiltration of subcutaneous tissues with no bone involvement (Fig. 1). The remainder of the eye examination and her general physical examination were unremarkable. Laboratory investigations including blood cell counts, thyroid function and serum IgE were normal. We had no information on the exact nature of the multiple eye operations done in Russia or what their diagnosis was. She underwent debulking surgery of the extraconal tumoral mass in her left eye. Due to progression of Kimura's disease, severe lagophthalmos and
IRIS MOROZ, NACHUM ROSEN, MORDECHAI ROSNER severe exposure keratitis, she underwent temporal tarsorrhaphy. Two years later she underwent debulking surgery of a left lower lid mass with a tightening procedure. At her last follow-up, swelling of the left upper eyelid was noted again.

The histopathological findings included prominent lymphoid follicles with germinal centres, proliferating capillaries lined with plump endothelial cells and abundant eosinophilic infiltration with lymphocytes and plasma cells, consistent with Kimura's disease (Fig. 2).

\section{Discussion}

Kimura's disease is a chronic inflammatory disease manifested by nodular lesions involving connective tissues that occurs predominantly in Orientals. It was first described in China by Kim and Szeto in 1937, and was known as Kimura's disease after Kimura, Yoshimura and Ishikawa who reported a similar condition in 1948 in Japan. ${ }^{1-3}$ Wells and Whimster in 1969 first reported patients in the West with a similar condition which they called angiolymphoid hyperplasia with eosinophilia (ALHE). Kimura's disease and ALHE have been regarded both as different presentations of one disease and as different disease entities. Batsakis and Manning suggested in 1986 that they are 'a fundamental biologic reaction with different clinical and pathological expressions in different races' ${ }^{4}$ Others suggest that they are different clinicopathological entities. ${ }^{1,3}$

Kimura's disease occurs predominantly in Chinese and Japanese, in a younger age group and the duration of the disease is longer than ALHE; the lesions are large masses of deep soft tissue without distinct borders. ALHE occurs in older patients and presents for a short duration; the lesions are small erythematous papules or nodules that itch and bleed easily.

The aetiology is not fully understood. An immunological disturbance or an allergic reaction was suggested in Kimura's disease, while ALHE was considered to be a proliferative reaction of vascular endothelial cells with a secondary inflammatory response. 

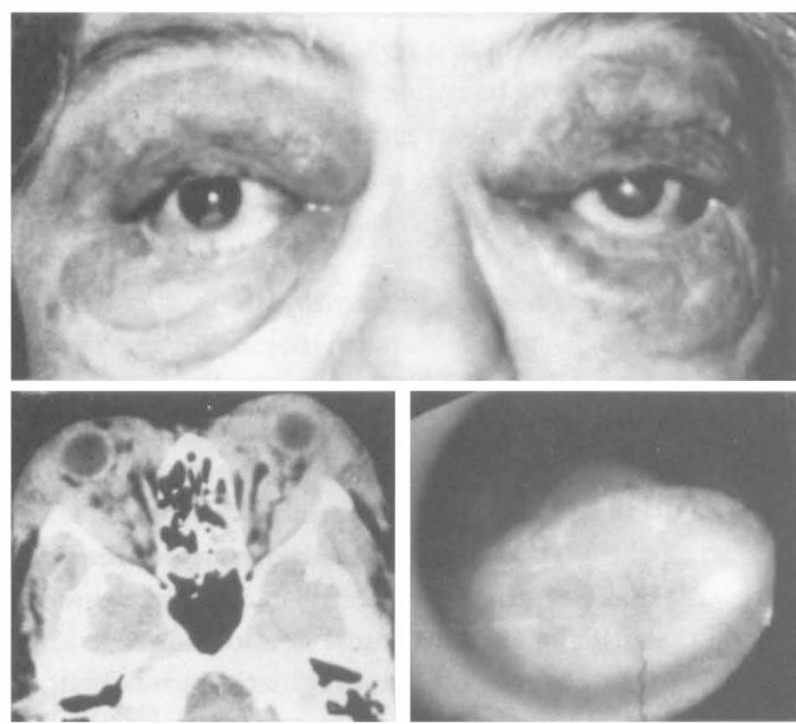

Fig. 1. Above: The patient at presentation, with bilateral exophthalmos, swollen eyelids and dark pigmentation. Below left: CT scan showing diffuse enlargement of the extraocular muscles and diffuse infiltration of the subcutaneous tissues. Below right: Corneal opacities of the left eye.
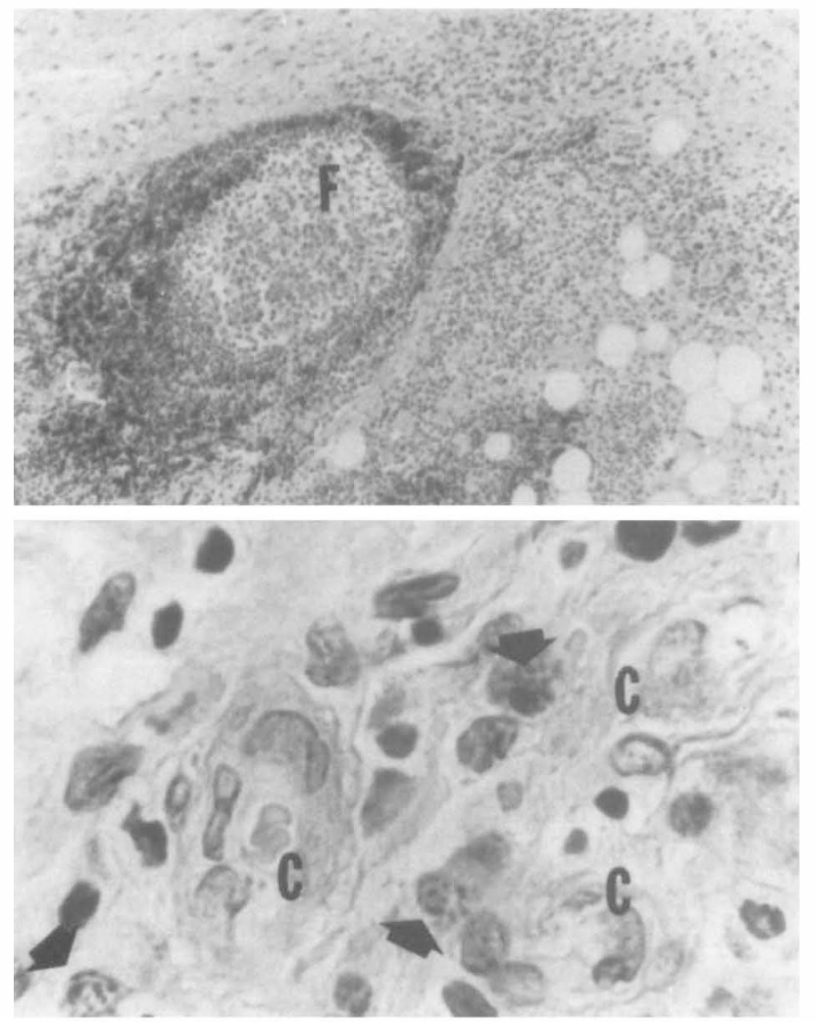

Fig. 2. Above: Histopathological section, at low magnification, of an orbital specimen demonstrating a prominent lymphoid follicle with germinal centre $(F)$ and diffuse leucocytic infiltration (haematoxylin and eosin, original magnification $\times 40)$. Below: High magnification of the same specimen demonstrating proliferating capillaries $(C)$ with plump endothelial cells and eosinophilic infiltrate (arrow) (haematoxylin and eosin, original magnification $\times 400$ ).

The lesions have a predilection for the head and neck. Kimura's disease occurs more often around the ear and salivary glands. Other sites such as the oral cavity, groin, trunks and limbs may also be involved. A search of the English literature revealed 18 cases of periocular involvement. ${ }^{1-7}$ All reported orbital cases were extraconal, poorly circumscribed, and grew in the anterior and superior orbital aspects along the contour of the globe and the bony walls. One case was associated with an Iowa implant after enucleation. ${ }^{7}$ Lymph node involvement in Kimura's disease has been reported to range from $67 \%$ to $100 \% .^{2}$ Blood eosinophilia and elevated IgE were more frequently seen in Kimura's disease than in ALHE. Malignant transformation was not noted in any of the cases of either entity. Systemic associations include asthma and nephrotic syndrome. The treatment of choice is surgical excision. Recurrence rate is $15^{\mathrm{a}} 40 \%$ due to the branching vascular nature of the condition that makes margins difficult to determine. ${ }^{1-3}$ Steroid treatment causes a rapid shrinkage of the lesion, but the action is transient and relapse is common after reduction or cessation of the drug. ${ }^{8}$ Radiotherapy treatment has good results with a decrease in size, but was not recommended for a benign condition. ${ }^{8}$

Our case had findings characteristic of both Kimura's disease and ALHE. As in Kimura's disease the patient had multiple subcutaneous nodules, and the onset was insidious at a young adult age. The histological findings were also characteristic of Kimura's disease. However, as seen in ALHE, our patient was Caucasian, and had no adenopathy, blood eosinophilia or raised serum IgE.

In conclusion, periocular Kimura's disease may have devastating outcome with deformed appearance and visual loss resulting from exposure keratitis. When the orbit is diffusely involved complete surgical excision is unattainable and progress of the disease is inevitable.

\section{References}

1. Chan JKC, Hui PK, Ng CS, Yuen NWF, Kung ITM, Gwi E. Epithelioid haemangioma (angiolymphoid hyperplasia with eosinophilia) and Kimura's disease in Chinese. Histopathology 1989;15:557-74.

2. Kuo T, Shih LY, Chang HL. Kimura's disease involvement of regional lymph nodes and distinction from angiolymphoid hyperplasia with eosinophilia. Am J Surg Pathol 1988;12:843-54.

3. Nyrop M. Kimura's disease: case report and brief review of the literature. J Laryngol Otol 1994;108:1005-7.

4. Batsakis JG, Manning JT. Soft tissue tumors: unusual forms. Otolaryngol Clin North Am 1986;19:659-83.

5. Hidayat AA, Cameron JD, Font RL, Zimmerman LE. Angiolymphoid hyperplasia with eosinophilia (Kimura's disease) of the orbit and ocular adnexa. Am J Ophthalmol 1983;96:176-89.

6. Kennedy SM, Pitts JF, Lee WR, Gibbons DC. Bilateral Kimura's disease of the eyelids. Br J Ophthalmol 1992;76:755-7.

7. Smith DL, Kincaid MC, Nicolitz E. Angiolymphoid hyperplasia with eosinophilia (Kimura's disease) of the orbit. Arch Ophthalmol 1988;106:793-5.

8. Tham KT, Leung PC, Saw D, Gwi E. Kimura's disease with salivary gland involvement. Br J Surg 1981;68:495-7. 